\title{
ABO Blood Groups and Severity to Plasmodium Falciparum
}

\section{Malaria Infections}

\author{
Barua $\mathbf{P}^{*}$ \\ Department of Zoology, University of Dhaka, Bangladesh
}

*Corresponding author: Priyanka Barua, Lecturer, Department of Zoology, University of Dhaka, Bangladesh, Email: baruap@du.ac.bd
Editorial

Volume 1 Issue 4

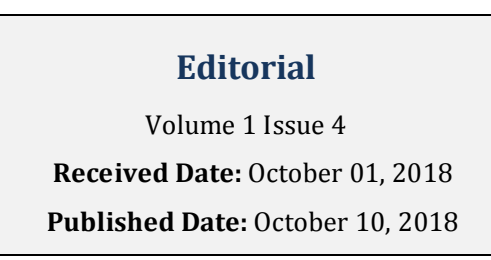

\section{Editorial}

Malaria is a leading cause of morbidity and mortality in pregnant women and children worldwide. This vectorborne disease is transmitted by female anopheline mosquitoes and caused by five species of the genus Plasmodium; Plasmodium falciparum, $P$. vivax, $P$. ovale, $P$. malariae, $P$. knowlesi. Among these disease-causing parasites, $P$. falciparum is the most virulent species causing the highest number of deaths specially in regions of Sub Saharan Africa and parts of Oceania with much higher transmission intensities than in most of Asia, South and Central America. According to World malaria report 2017, P. falciparum caused $99 \%$ of the estimated malaria cases in 2016 within Sub Saharan African region. P. falciparum related malaria results in severe clinical complications i.e. severe malaria by the sequestration of parasitized red blood cells (RBC) in vital organs such as brain, lung, heart etc. The severity of falciparum malaria has also been associated with the adhesion of infected RBCs to the uninfected ones; a mechanism known as rosetting. As the pathological complexity of severe malaria is dependent on the RBC sequestration, it is necessary to understand whether different $\mathrm{ABO}$ blood group types are related to the differences in severity and susceptibility to malarial infections.

Several previous studies have noted the differences in the severity of malarial infections according to different ABO blood types. In general, studies from malaria endemic countries showed that the severity of infection is more prominent in non-O blood groups than blood group 0 patients. Clinical manifestations of severe malaria have been more commonly observed in blood group A patients while blood group $O$ have been associated with protection from severe malaria. One of the proposed reasons behind the less severity of malaria in blood group 0 individuals could be the lower rate of rosetting in blood group 0 than in the other blood types. The A and B antigen structure differs from blood group 0 individuals as the trisaccarides in A and B antigens can operate as receptors responsible for rosetting. This structural difference may lead to the contrasting rate of rosetting between blood group 0 and non- $\mathrm{O}$ patients ultimately resulting in differences in severity.

Moreover, P. falciparum parasite has a unique mechanism of cytoadhesion by the expression of an adhesive protein on the surface of the infected RBCs known as $\mathrm{P}$ falciparum erythrocyte membrane protein-1 (Pf EMP1). Pf EMP1 is encoded by highly polymorphic gene family called var genes. Although $\sim 60 \mathrm{var}$ genes exist in parasite genome, only single of them expresses at a time. The typical structure of PfEMP1 includes: the variable $\mathrm{N}$-terminal segment (NTS), variable numbers of Duffy binding like domains (DBL, $\alpha-\varepsilon$ ), one or two cysteine-rich inter domain regions (CIDR, $\alpha-\gamma$ ), a trans membrane domain (TM), a $\mathrm{C} 2$ domain and a conserved intra-cellular acidic terminal segment (ATS). The specific Pf EMP1 adhesion which causes rosetting has a typical DBL1 $\alpha 1-C I D R 1 \gamma$ domain structure. Preferential binding of Var $\mathrm{O}$ rosette causing parasite has been shown with blood group A than non-O blood types (B and O). This implies that $A$ and $B$ antigens itself act as binding ligands for rosetting. In the presence of $A$ and $B$ antigens, infected RBCs can adhere with uninfected RBCs causing cytoadhesion and increasing the severity of the disease. The association between rosetting, ABO blood types and PfEMP1 expression in severe malaria needs further exploration as it is still a comparatively understudied 


\section{International Journal of Zoology and Animal Biology}

area. Further research in this field may help to find out how certain RBC polymorphism may aid in conferring

protection against severe malaria.

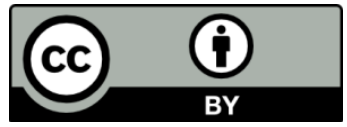

